\title{
Experimental Determination of the Mean Free Path of Screened Edge Magnetoplasmons in a Two-Dimensional Electron Gas
}

\author{
V. M. Muravev ${ }^{a, b}$, I. V. Andreev ${ }^{a}$, I. V. Kukushkin ${ }^{a, b}$, J. H. Smet ${ }^{b}$, and K. von Klitzing ${ }^{b}$ \\ ${ }^{a}$ Institute of Solid State Physics, Russian Academy of Sciences, Chernogolovka, Moscow region, 142432 Russia \\ ${ }^{b}$ Max-Planck-Institut für Festkörperforschung, Stuttgart, 70569 Germany \\ Received April 17, 2008
}

\begin{abstract}
Magnetic oscillations of the photovoltage in a two-dimensional electron system with the back gate, exposed to microwave radiation, are studied. The oscillations result from the interference of screened edge magnetoplasmons (EMPs). The mean free path of the EMPs is quantitatively determined by analyzing the dependence of the oscillation amplitude on the electron density. The dependences of the mean free path of the EMPs on the two-dimensional electron density, microwave frequency, electron relaxation time, and the magnetic field are studied. It is found that the dependences agree qualitatively with the known theoretical calculations.
\end{abstract}

PACS numbers: 67.57.Lm, 76.60.-k

DOI: $10.1134 / \mathrm{S} 0021364008100135$

Plasma excitations in two-dimensional electron systems have been intensively studied for over 30 years $[1,2]$, in particular, because many parameters of plasma waves, contrary to, e.g., optical or electronic waves, can be easily controlled by varying the electron density or by applying an external magnetic field. This makes plasmons flexible and convenient objects for physical studies and various applications. One of the main manifestations of the wave nature of plasmons is their interference. The phenomenon of plasmon interference has not been experimentally observed for a long time because of the low quality of available structures. Significant progress in the structure fabrication technologies achieved in the last decade has led, in particular, to a significant increase in the two-dimensional electron mobility. As a result, a new type of magnetic oscillation of the photovoltage and longitudinal magnetoresistance in high-mobility two-dimensional electron systems exposed to microwave radiation has recently been discovered and studied [3, 4]. The effect was caused by the interference of edge magnetoplasmons (EMPs), which were coherently excited by the electromagnetic radiation in near-contact regions and propagated along the boundary of the two-dimensional electron gas. The magneto-oscillation period turned out to be determined by the radiation frequency, electron-gas density, and distance between the potential contacts along the edge of the electron system. These features open unique possibilities for applying the effect of the EMP interference for the detection and spectroscopy of microwave and terahertz radiation $[5,6]$.

The effect of the EMP interference can be observed if the plasmon mean free path exceeds distance $L$ from the place of plasmon excitation to the place of interference. Therefore, studying the mean free path of the EMPs is an important issue. The first experiments on the determination of the plasmon mean free path [7] showed that it can amount to a few millimeters in electron systems with mobility $\mu=10^{6} \mathrm{~cm}^{2} / \mathrm{V} \mathrm{s}$. This makes it possible to observe and study the effect of the plasmon interference in macroscopic (millimeter) structures. However, although the dependence of the mean free path of the EMPs on the two-dimensional electron density, microwave frequency, electron mobility, and magnetic field is crucial for choosing conditions favoring the observation of the plasmon interference, no attempts were undertaken until recently to study this dependence in detail. In this paper, the behavior of the amplitude of microwave-induced magnetic oscillations in a structure was studied for various electron densities controlled by a back gate. As a result, we succeeded in measuring the mean free path of the screened EMPs and its dependence on the two-dimensional electron density, magnetic field, and microwave frequency.

The studied structure was a single GaAs/AlGaAs quantum well with a width of $18 \mathrm{~nm}$ located at a depth of $135 \mathrm{~nm}$ from the crystal surface. An $n^{+}$GaAs back gate has been grown under the quantum well at a distance of $765 \mathrm{~nm}$ to provide for controlled variations in the two-dimensional electron gas density. The electron density and mobility were varied from $0.7 \times 10^{11}$ to $2.5 \times 10^{11} \mathrm{~cm}^{-2}$ and from $0.7 \times 10^{6}$ to $2.1 \times 10^{6} \mathrm{~cm}^{2} / \mathrm{V} \mathrm{s}$, respectively. A mesa in the form of a Hall bridge with the sizes and geometry used in our previous experimental works [3, 7] was formed lithographically on the structure. The sample fixed at the end of a 16-mm 


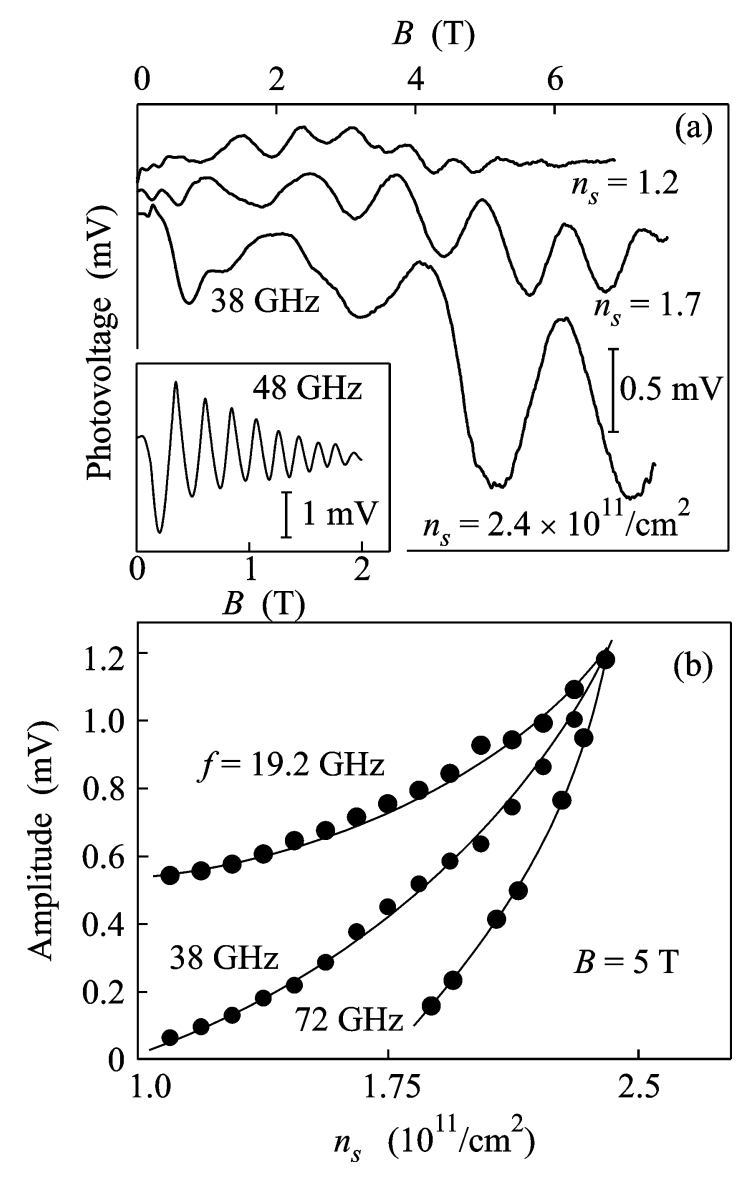

Fig. 1. (a) Photovoltage oscillations measured for the microwave frequency $f=38 \mathrm{GHz}$ in a structure with $L=$ $50 \mu \mathrm{m}$ for three different electron densities. For convenience, the plots are spaced apart vertically. The inset shows a plot of magnetic oscillations of the photovoltage for a structure with $L=200 \mu \mathrm{m}$ and $n_{s}=1.8 \times 10^{11} \mathrm{~cm}^{-2}$ and for $f=48 \mathrm{GHz}$. (b) The amplitude of the photovoltage oscillations in the magnetic field $B=5 \mathrm{~T}$ versus the electron density for the microwave frequencies $f=19.2,38$, and $72 \mathrm{GHz}$.

waveguide was placed in a helium cryostat inside a superconducting solenoid. The microwave radiation was input to the sample via this waveguide. The microwave oscillators covered the frequency band from 19 to $80 \mathrm{GHz}$, and the output power was not higher than $10 \mathrm{~mW}$. A technique for the synchronous detection of the photovoltage signal for a $1-\mathrm{kHz}$ modulation of the incident radiation was used for the measurements. The experiments were carried out using a sample at a liquid helium temperature of $4.2 \mathrm{~K}$.

Figure 1a shows typical experimental magneticfield dependences of the photovoltage measured for various two-dimensional electron densities and for the microwave frequency $f=38 \mathrm{GHz}$ and the length $L=$ $50 \mu \mathrm{m}$, as well as for $f=48 \mathrm{GHz}, L=200 \mu \mathrm{m}$, and the electron density $n_{s}=1.8 \times 10^{11} \mathrm{~cm}^{-2}$. It is seen that these curves are $B$-periodic oscillations whose amplitude increases with the electron density. Such a form for the magnetic-field dependences of the photovoltage is explained by the interference of screened EMPs coherently excited in the near-contact regions of the studied structure [3]. Edge magnetoplasmons (EMPs) belong to a special type of plasma waves propagating along the boundary of a two-dimensional electron system in sufficiently strong magnetic fields $\left(\omega_{c} \tau>1\right.$, where $\omega_{c}$ is the cyclotron frequency and $\tau$ is the time of the elastic electron relaxation). The velocity of screened EMPs is proportional to the Hall conductivity [8]:

$$
V_{\mathrm{emp}} \propto \sigma_{x y} \propto n_{s} e^{2} / m^{*} B,
$$

where $n_{s}$ is the two-dimensional electron density, $B$ is the magnetic field, and $e$ and $m^{*}$ are the electron charge and effective mass, respectively. Using Eq. (1), it is easy to find the dependence of the period $\Delta B$ of photovoltage magnetic oscillations on the frequency $f$ of the incident radiation, two-dimensional electron density, and distance $L$ from the point of excitation to the point of interference of the screened EMPs. Setting the EMP wave vector equal to $2 \pi N / L$ in the case of constructive interference, where $N$ is an integer, we find the magnetic-oscillation period $\Delta B \propto n_{s} / f L$. The electron-density dependence of the amplitude of the photovoltage oscillations in the magnetic field $B=5 \mathrm{~T}$ for various microwave frequencies is shown in greater detail in Fig. 1b. The curves for each of these three frequencies are normalized to the same oscillation amplitude corresponding to the electron density $n_{s}=2.4 \times 10^{11} \mathrm{~cm}^{-2}$. It is seen that the amplitude of the photovoltage oscillations increases with the density, and the amplitude variations are more pronounced at higher microwave frequencies.

To determine the mean free path of the EMPs $L_{\mathrm{emp}}$, we write the amplitude of the magnetic oscillations of the photovoltage $U$ in terms of $L_{\text {emp }}$. Since the amplitudes of the emitted wave and wave passed the distance $L$ are summed at the interference point near the contact, the voltage $U_{r}$ at the contact due to the quadratic nonlinearity and rectification is equal to

$$
U_{r}=U_{0}\left|1+e^{-i q L}\right|^{2},
$$

where $q=q_{1}-i q_{2}, q_{1} \propto \omega B / n_{s}$ is the plasmon wave vector, and the term $q_{2}=1 / L_{\mathrm{emp}}$ is responsible for its decay. Reduction of Eq. (2) yields

$$
U_{r}=U_{0}\left(1+e^{-2 q_{2} L}+2 e^{-q_{2} L} \cos q_{1} L\right) .
$$

According to Eq. (3), the amplitude of the magnetic oscillations of the photovoltage decreases with $L_{\mathrm{emp}}$ as

$$
U \propto e^{-q_{2} L}=e^{-L / L_{\mathrm{emp}}} .
$$

Now, it is easy to understand the qualitative behavior of the experimental curves in Fig. 1. First of all, this figure shows that the amplitude of the photovoltage oscillations increases with the electron density $n_{s}$; according to Eq. (4), this behavior is explained by the 
increase in $L_{\mathrm{emp}}$ with an increase in $n_{s}$. In addition, it is easily seen that, according to the inset in Fig. 1a, the oscillation amplitude decreases with an increase in the magnetic field $B$, because $L_{\text {emp }}$ decreases with an increase in $B$. The determination of the relation between the mean free path of the EMPs and the microwave frequency $f$ is more complicated. According to Fig. 1b, the amplitude of the photovoltage oscillations becomes a steeper function of the electron density as the radiation frequency increases. Hence, the mean free path $L_{\text {emp }}$ decreases with an increase in $f$.

According to the theory developed in [9], the mean free path of the EMPs along the edge of a semi-infinite electron system is given by

$$
L_{\mathrm{emp}} \propto \frac{n_{s} e^{2} \tau}{m^{*} \omega} \frac{1}{1+0.09 \frac{\omega_{\mathrm{c}} \tau}{\sqrt{1+\omega^{2} \tau^{2}}}},
$$

where $\omega_{c}=e B / m^{*}$ is the cyclotron frequency. The above theoretical relation was obtained in the approximation that the electron gas occupies the interface between the dielectric substrate and a vacuum. A comparison of theoretical formula (5) and the above analysis of the experimental plots in Fig. 1 shows that the theory correctly describes the qualitative behavior of the mean free path of the EMPs $L_{\text {emp }}$ in low magnetic fields as a function of various parameters. To determine the value of $L_{\text {emp }}$, we show the plasmon mean free path is proportional to the electron-gas conductivity in a zero magnetic field: $L_{\mathrm{emp}} \propto \sigma_{0}=n_{s} e^{2} \tau / m^{*}$. To this end, we measure the amplitude of the magnetic oscillations of the photovoltage $U$ as a function of the electron density $n_{s}$ for the fixed magnetic field $B=5 \mathrm{~T}$ (see Fig. 1b) and evaluate the electron-system resistivity $\rho_{0}=1 / \sigma_{0}$ in the zero magnetic field for each $n_{s}$ value (see Fig. 2a). Taking into account Eq. (4) and assuming that $L_{\text {emp }} \propto$ $\sigma_{0}$, we arrive at the following expression for the logarithm of the amplitude of photovoltage oscillations:

$$
\ln U=A-L / L_{\mathrm{emp}}=A-C(\omega, B) L \rho_{0},
$$

where $\rho_{0}=1 / \sigma_{0}, A$ is a constant, and $C(\omega, B)$ is a function of the radiation frequency and magnetic field. The experimental data perfectly confirm that the plasmon mean free path is proportional to the electron-gas conductivity. Indeed, Fig. 2a shows the plots $\ln U\left(\rho_{0}\right)$ for two microwave frequencies 19.2 and $38 \mathrm{GHz}$ and a fixed magnetic field of $5 \mathrm{~T}$. According to Eq. (6), these dependences are linear, and the slopes of these lines are given by $C(\omega, B) L$. Since $L=50 \mu \mathrm{m}$ for the structure used in the experiments, the function $C(\omega, B)$ can be evaluated from the slop of the line and, therefore, the mean free path of the EMPs $L_{\text {emp }}$ can be found for any $\rho_{0}$. Thus, the behavior of $L_{\text {emp }}$ as a function of the twodimensional electron density $n_{s}$ can be found quantitatively (see Fig. 2b). This dependence is linear in agreement with theoretical prediction (5). According to the
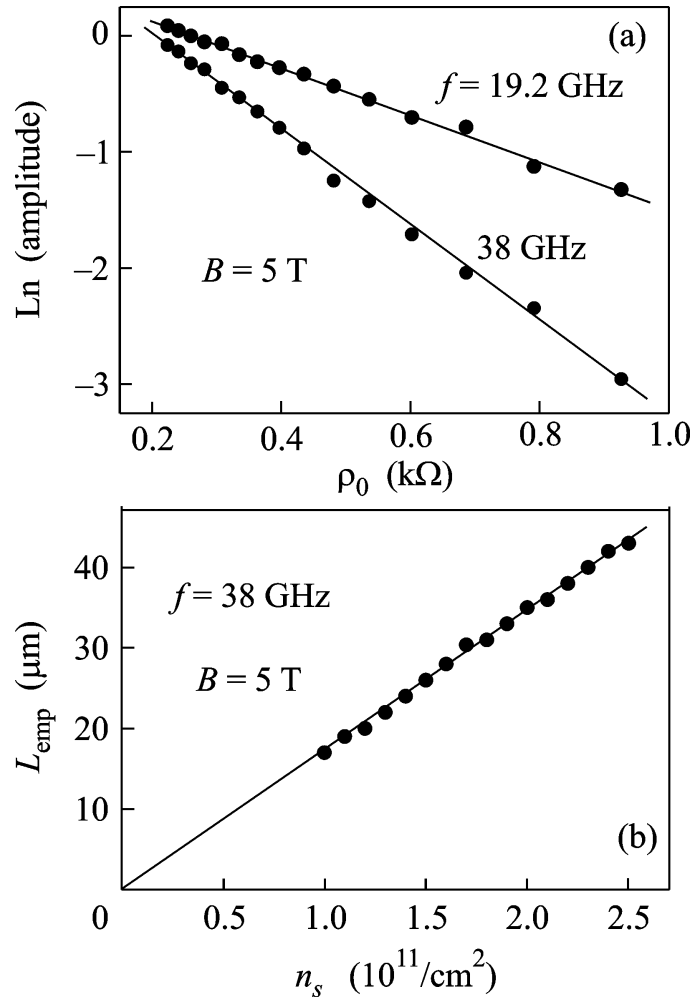

Fig. 2. (a) The logarithm of the amplitude of the photovoltage oscillations versus the electron-gas resistivity for two microwave frequencies. The resistivity $\rho_{0}$ was controlled by varying the electron density by virtue of the back gate. (b) The mean free path of the screened edge magnetoplasmons versus the two-dimensional electron density for the microwave frequency $f=38 \mathrm{GHz}$ and the magnetic field $B=5 \mathrm{~T}$. The data were obtained for a sample at the temperature $T=4.2 \mathrm{~K}$.

obtained data, the mean free path of the EMPs for a microwave frequency of $38 \mathrm{GHz}$ and an electron density of $1.7 \times 10^{11} \mathrm{~cm}^{-2}$ is $L_{\text {emp }}(B=5 \mathrm{~T})=30 \mu \mathrm{m}$.

To determine the dependence of the mean free path of the screened EMPs on the microwave frequency $f$ and magnetic field $B$, the procedure described above was carried out for various frequencies and magnetic fields. The results are summarized in Fig. 3. The experiment shows that the mean free path of the screened EMPs $L_{\text {emp }}$ is inversely proportional to the frequency $f$ (Fig. 3b) and decreases slightly with an increase in the magnetic field (Fig. 3a). The plasmon mean free path as a function of the magnetic field is described best of all by the formula

$$
L_{\mathrm{emp}}=\frac{L_{0}}{1+\left(B / B_{0}\right)^{2}},
$$

where $B_{0}=6 \mathrm{~T}$ and $L_{0}=41 \mu \mathrm{m}$ for $f=52.5 \mathrm{GHz}$. Some discrepancy between experimental dependence (7) and theory (5) can be explained by the difference of the architectures of the structures used in experiments and considered theoretically. 


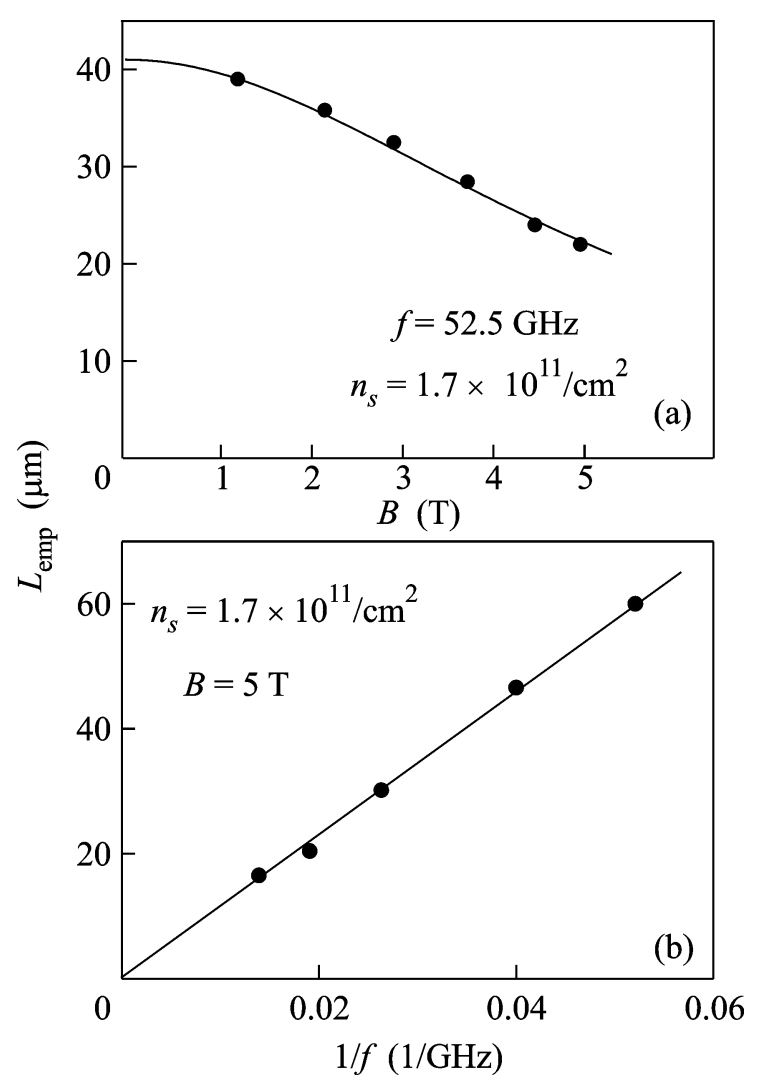

Fig. 3. (a) The mean free path of screened edge magnetoplasmons versus the magnetic field for the microwave frequency $f=52.5 \mathrm{GHz}$ and the two-dimensional electron density $n_{s}=1.7 \times 10^{11} \mathrm{~cm}^{-2}$. (b) The mean free path of screened edge magnetoplasmons versus the microwave frequency for the two-dimensional electron density $n_{s}=1.7 \times 10^{11} \mathrm{~cm}^{-2}$ and the magnetic field $B=5 \mathrm{~T}$. The data were obtained for a sample at the temperature $T=4.2 \mathrm{~K}$.

It was shown in our earlier study [7] that the mean free path of the EMPs is proportional to the electron relaxation time $\tau$ and amounts to $L_{\text {emp }}(T=4.2 \mathrm{~K})=$ $0.5 \mathrm{~mm}$ for an incident-radiation frequency of $46 \mathrm{GHz}$ and the electron density $n_{s}=2.6 \times 10^{11} \mathrm{~cm}^{-2}$. These results were obtained for a two-dimensional electron system without a back gate. As shown above, the presence of a metallic gate at the distance $d=765 \mathrm{~nm}$ from the electron system significantly reduces the plasmon mean free path to $70 \pm 2 \mu$ m under the same conditions. To clarify the correspondence between these two experiments, we note that, according to [10], the velocity of the plasma waves in an electron system decreases significantly in the presence of a nearby back gate. This explains the significant decrease in the plasmon mean free path observed for structures with the back gate.

It should be noted that the reported study is very important for various applications based on the features of the plasma excitations in low-dimensional electron systems $[11,5,12]$. The results allow one to predict the operating parameters of a device as functions of the temperature and radiation frequency.

Thus, an experimental technique for the quantitative determination of the mean free path of screened edge magnetoplasmons is developed in this work. The technique is applied to analyze the dependence of the mean free path of screened magnetoplasmons on the twodimensional electron density, microwave frequency, electron relaxation time, and magnetic field. The results are found to agree qualitatively with current theoretical calculations.

We are grateful to V.A. Volkov and S.A. Mikhailov for helpful discussions and comments. This work was supported by the Russian Foundation for Basic Research and Deutsche Forschungsgemeinschaft. V.M.M. acknowledges the support of Forschungszentrum Jülich through a Landau Scholarship.

\section{REFERENCES}

1. D. Heitmann, Surf. Sci. 170, 332 (1986).

2. V. A. Volkov and S. A. Mikhailov, in Landau Level Spectroscopy, Ed. by G. Landwehr and E. I. Rashba (Elsevier, Amsterdam, 1991), Chap. 15, p. 855.

3. I. V. Kukushkin, M. Yu. Akimov, J. H. Smet, et al., Phys. Rev. Lett. 92, 236803 (2004).

4. B. Simovič, C. Ellenberger, K. Ensslin, et al., Phys. Rev. B 51, 13467 (2005).

5. I. V. Kukushkin, S. A. Mikhailov, J. H. Smet, et al., Appl. Phys. Lett. 86, 044101 (2005).

6. P. S. Dorozhkin, S. V. Tovstonog, S. A. Mikhailov, et al., Appl. Phys. Lett. 86, 044101 (2005).

7. V. M. Muravev, I. V. Kukushkin, A. L. Parahonskii, et al., JETP Lett. 83, 290 (2006).

8. V. A. Volkov and S. A. Mikhailov, JETP 94, 217 (1988).

9. S. A. Mikhailov, Appl. Phys. Lett. 89, 042109 (2006).

10. V. M. Muravev, C. Jiang, I. V. Kukushkin, et al., Phys. Rev. B 75, 193307 (2007).

11. W. Knap, Y. Deng, S. Rumyantsev, et al., Appl. Phys. Lett. 80, 18 (2002).

12. E. A. Shaner, M. Lee, M. C. Wanke, et al., Appl. Phys. Lett. 87, 193507 (2005).

Translated by A. Serber 